\title{
The VERA project (VLBI Exploration of Radio Astrometry)
}

\author{
Hideyuki Kobayashi ${ }^{1}$, Noriyuki Kawaguchi ${ }^{1}$, Seiji Manabe ${ }^{1}$, \\ Toshihiro Omodaka ${ }^{2}$, Katsunori Shibata ${ }^{1}$, Mareki Honma ${ }^{1}$, \\ Yoshiaki Tamura ${ }^{2}$, Osamu Kameno ${ }^{2}$, Tomoya Hirota ${ }^{2}$, Hiroshi Imai ${ }^{1}$ \\ ${ }^{1}$ National Astronomical Observatory of Japan, Mitaka, Tokyo, 181-8588, Japan \\ 2 Department of Physics, Kagoshima University, Kagoshima, Japan
}

\begin{abstract}
VLBI - Very Long Baseline Interferometry - is a radio interferometry technique which provides the highest spatial resolution observations to human kind. But at the present, the accuracy of the astrometry observations is limited by the atmospheric light path variations and instrumental phase errors, and only group delay measurements are used. To overcome these error factors, we have developed the VERA system, which has the first dual beam system. VERA is the first VLBI array to be free from the atmospheric phase fluctuations. It has four VLBI stations with 2300-km maximum baseline length in Japan. To compensate phase fluctuations of interferometer visibilities, which are mainly caused by the atmosphere, the VERA antenna observes two objects simultaneously. In order to do such observations, VERA has a two-receiver system, which tracks a focal plane according to a separation angle between observing objects. By comparing the visibility phase between two beams, simultaneous phase referencing VLBI will be achieved. The goal accuracy of astrometry observations is 10 micro-arcseconds, which makes annual parallax and proper motion measurements of galactic maser objects possible. 10 microarcsecond accuracy is equivalent to $10 \%$ distance accuracy for the galactic centre. This becomes $20 \%$ accuracy at the opposite side of the galaxy. The main scientific targets of VERA are to make a 3-dimensional maser object map of the galaxy and reveal the velocity field of the galaxy. This will show the mass distribution of the galaxy. Currently, construction of four stations is complete, and test observations are underway. We show the scientific goal, current results and instrumental accuracy of VERA.
\end{abstract}

ED BudDing: Could you give some idea about the limiting flux density of point sources that you can receive with VERA? Is it something like $0.1 \mathrm{mJy}$ ?

Hideyuki KoBAyAshi: For the VLBI observations the structure is omitted, because we use only the interferometer. So it is only sensitive for very compact structures and we need some correction for the structure effect. We want to know the mapping, especially for the calibrator - from VLBI, or from ourselves.

ED BudDing: The contours on the map that you showed: they are what kind of fluxes in mJy?

Hideyuki Kobayashi: Its a mJy per bin, and the bin is very small. This shows very high temperature regions; the usual extended structure vanished. 